\title{
Childhood Cerebral Diffuse Astrocytoma
}

National Cancer Institute

\section{Source}

National Cancer Institute. Childhood Cerebral Diffuse Astrocytoma. NCI Thesaurus. Code C8387.

A diffuse astrocytoma that arises from the cerebral hemispheres and occurs during childhood. 